\title{
Fluxo de capitais, diferencial de juros e o modelo de crescimento com restrição no balanço de pagamentos: uma perspectiva teórica
}

Capital flow, interest rate differential, and balance of payments constraint growth model: a theoretical perspective

Marwil J.Dávila-Fernández

Università degli Studi di Siena

\begin{abstract}
This paper aims to contribute to the debate on long-term economic growth, clarifying aspects of the balance of payments constraint growth models. We formalized a theoretical model in which foreign direct investment is distinguished from portfolio investment in determining the external constraint growth rate, so that part of the attraction of foreign capital results from the differential rates of domestic and foreign interest and a risk premium.
\end{abstract}

\section{Keywords}

economic growth; balance of payments constraint; Thirlwall's Law.

JEL Codes O11; P16.

\section{Resumo}

O trabalho tem como objetivo contribuir para o debate em torno do crescimento economico de longo prazo, esclarecendo aspectos dos modelos de crescimento com restrição no Balanço de Pagamentos (BP). Formalizamos um modelo teórico em que o investimento externo direto é diferenciado do investimento em carteira na determinação da taxa de crescimento compativel com a restrição externa, de modo que parte do fluxo de capitais decorre do diferencial das taxas de juros doméstica, internacional e do prêmio de risco.

\section{Palavras-chave}

crescimento econômico; balanço de pagamentos; Lei de Thirlwall.

Códigos JEL O11; P16. 


\section{Introdução}

O problema do crescimento é tão complexo quanto fascinante. Utilizando uma abordagem neoclássica, os primeiros trabalhos nessa literatura centraram suas conclusões nos fatores de produção que compõem a função de produção, tratando o progresso técnico como exógeno ao sistema econômico. Nas últimas duas décadas, a teoria do crescimento endógeno apenas reforçou o papel da função de produção, modelando agora o capital humano, mas mantendo a orientação suply-side de seus resultados (Setterfield, 2011a). Alternativamente, a escola pós-keynesiana desenvolveu uma série de contribuições dando ênfase especial ao papel da demanda no processo de crescimento das economias capitalistas.

Dentre os modelos demand-side, sobressaem-se os chamados balance-of-payments-constrained (BOPC), notadamente representados pela Lei de Thirlwall. Sua proposição central está em que, para a maioria dos países, a principal restrição à taxa de crescimento do produto está no Balanço de $\mathrm{Pa}$ gamentos (BP) porque ele determina o limite do crescimento da demanda a que a oferta pode se adaptar (Thirlwall; Hussain, 1982; Alonso; Garcimartín, 1999; Thirlwall, 2011). Os modelos BOPC têm sido corroborados empiricamente em uma série de estudos tanto em países desenvolvidos quanto em desenvolvimento. Em sua versão original a Lei de Thirlwall postula que a taxa de crescimento que equilibra o Balanço de Pagamentos no longo prazo não pode ser superior ao produto da (i) razão entre as elasticidades das exportações e importações e (ii) a taxa de crescimento do resto do mundo.

Dito isso, este trabalho tem como objetivo contribuir para o debate em torno do crescimento econômico de longo prazo, esclarecendo aspectos dos modelos de crescimento com restrição externa. Apresentaremos a evolução da modelagem em torno da conhecida Lei de Thirlwall, centrando-nos em autores que procuram incorporar na expressão original de 1979 os demais elementos do Balanço de Pagamentos. Formalizamos ainda um modelo teórico em que o investimento externo direto é diferenciado do investimento em carteira na determinação da taxa de crescimento que equilibra o BP, de modo que parte da atração de capitais externos decorre do diferencial de taxas de juros doméstica, externa e do prêmio de risco.

A principal contribuição do trabalho para a literatura está na diferenciação dos fluxos de investimento de longo e curto prazos. Esse parece ser o último passo para termos todos os principais elementos do Balanço de $\mathrm{Pa}$ - 
gamentos no referencial BOPC. Mesmo assim, ainda ficaram de fora rubricas da conta Capital e Financeira como "Derivativos Financeiros" e "Outros Investimentos", que podem vir a ser incorporados em próximos trabalhos.

O artigo estrutura-se em quatro seções além desta introdução. A seção 2 apresenta a evolução histórica dos principais modelos de crescimento com restrição no Balanço de Pagamentos, desde Thirlwall até as recentes contribuições de Moreno-Brid e Alleyne e Francis. Em seguida, na seção 3, realizamos uma modificação no modelo de Moreno-Brid (2003) de modo a captar o efeito do diferencial da taxa de juros doméstica e externa sobre a taxa de crescimento que equilibra o BP. A seção 4 traz as considerações finais.

\section{Balanço de Pagamentos e o crescimento de longo prazo}

Um dos principais objetivos da teoria do crescimento tem sido explicar por que as taxas de crescimento variam tanto no tempo e entre países (Alonso; Garcimartín, 1999). Passados mais de duzentos anos desde a publicação de The Wealth of Nations, ainda não temos uma resposta conclusiva, muito embora tenham sido feitas importantes contribuições.

Utilizando uma abordagem neoclássica, os primeiros trabalhos em crescimento centraram suas conclusões nos fatores de produção que compõem a função de produção de uma economia. Mais recentemente, a teoria do crescimento endógeno apenas reforçou o papel da função de produção, mantendo a orientação suply-side de seus resultados.

Da mesma forma que Thirlwall e Hussain (1982), nossa argumentação começa pela proposição de que, para a maioria dos países, a principal restrição à taxa de crescimento do produto está no Balanço de Pagamentos porque ele determina o limite do crescimento da demanda a que a oferta pode se adaptar. Assumir que as restrições pelo lado da oferta são válidas só se sustenta sobre a hipótese neoclássica de uma economia com apenas um setor (Thirlwall, 1979; Alonso; Garcimartín, 1999; Thirlwall, 2011).

A ênfase na demanda como motor do sistema econômico enquanto as diferenças nas taxas de crescimento seriam fruto de distintos ritmos de crescimento da demanda não é nova na literatura, sendo trabalhada por economistas renomados como Kaldor, Prebisch, Thirlwall e McCombie. A hipótese fundamental dos modelos chamados balance-of-payments-constrained é que 
o Balanço de Pagamentos no longo prazo deve estar equilibrado. Diante da impossibilidade do financiamento contínuo dos desequilíbrios no BP, ocorre um ajuste da demanda agregada que restringe sua expansão e, por sua vez, o crescimento (Romero; Silveira; Jayme Jr, 2011; Setterfield, 2011b).

O Balanço de Pagamentos (BP) agrega as principais operações entre a economia doméstica e a internacional (Figura 1). Ele se subdivide na conta de "Transações Correntes" (CTC) e na conta "Capital e Financeira" (CCF). A primeira é formada pela "Balança Comercial" (BC), "Serviços e Rendas" (SR) e "Transferências" ( $\mathrm{T}$ ), sendo esta última importante especialmente para pequenas economias no Caribe e no continente africano. A segunda, por seu turno, é composta do "Investimento Externo Direto" (IED), o "Investimento em Carteira" (IC), "Derivativos Financeiros" e "Outros Investimentos". A compreensão dos componentes do BP é importante para o estudo dos modelos BOPCna medida em que a modelagem destes últimos parte de simplificações da sua estrutura.

\section{Figura 1 Componentes do Balanço de Pagamentos}

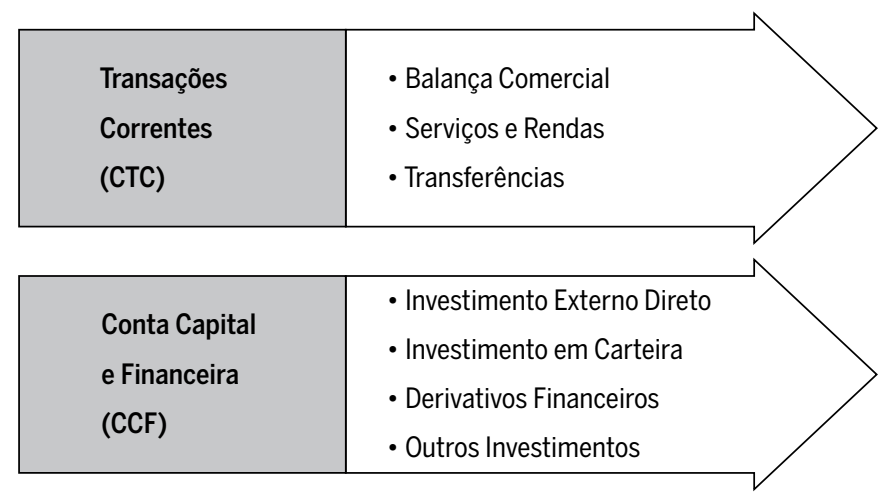

Fonte: Elaborado pelo autor.

A magnitude dos saldos da conta "Derivativos Financeiros" costuma ser modesta no caso dos países em desenvolvimento; contudo, isso não se aplica para a rubrica "Outros Investimentos", na qual se inclui, por exemplo, dívida externa e suas amortizações. No caso do Brasil, em 2013 o saldo da rubrica IC foi de US\$25.7 bilhões enquanto o saldo da rubrica "Outros Investimentos" foi de US\$ 19.9 bilhões, não sendo, portanto, um valor desprezível. Feita essa observação, o artigo considerará para efeito de simplificação apenas as rubricas IED e IC dentro da CCF. 


\subsection{0 modelo original de Thirlwall (1979)}

O modelo canônico de Thirlwall (1979) assume que o desempenho do Balanço de Pagamentos equivale ao da balança comercial. Dessa forma, podemos sumarizá-lo em três equações, sendo que nesta exposição a taxa de câmbio nominal é tomada fixa e igual à unidade:

$$
\begin{aligned}
& \mathrm{X}=\left(\frac{\mathrm{P}_{\mathrm{d}}}{\mathrm{P}_{\mathrm{f}}}\right)^{\varphi} \mathrm{Z}^{\Phi} \\
& M=\left(\frac{P_{f}}{P_{d}}\right)^{\beta} Y^{\pi} \\
& P_{d} X=P_{f} M
\end{aligned}
$$

onde $X$ representa as exportações; $M$, as importações; $Z$, a renda mundial; $Y$, a renda doméstica; $P_{d}$ corresponde ao nível de preços internos, e $P_{f}$, aos preços externos. Finalmente, $\varphi, \beta<0$ e $\Phi, \pi>0$ correspondem às elasticidades preço-demanda e renda-demanda das exportações e importações, respectivamente. A equação (3) corresponde à condição de equilíbrio no BP. Logaritmando e derivando no tempo:

$$
\begin{aligned}
& \hat{x}=\varphi\left(\widehat{p_{d}}-\widehat{p_{f}}\right)+\Phi \hat{z} \\
& \hat{m}=-\beta\left(\widehat{p_{d}}-\widehat{p_{f}}\right)+\pi \hat{y} \\
& \widehat{p_{d}}+\hat{x}=\widehat{p_{f}}+\hat{m}
\end{aligned}
$$

Substituindo (4) e (5) em (6) e isolando $\hat{y}$, obtemos a taxa de crescimento compatível com o equilíbrio no Balanço de Pagamentos:

$$
\hat{y}=\frac{(1+\varphi+\beta)\left(\widehat{p_{d}}-\widehat{p_{f}}\right)+\Phi \hat{z}}{\pi}
$$

Alonso e Garcimartín (1999) argumentam que não há evidência significativa da relação entre os preços relativos e o Balanço de Pagamentos. $\mathrm{Na}$ versão neoclássica do modelo de Thirlwall, os preços relativos estão em 
função das rendas interna e externa e também das elasticidades. ${ }^{1}$ Assumindo preços flexíveis no longo prazo, eles ajustariam eventuais desequilíbrios no BP. Entretanto, como apresentado pelos autores, essa posição não é corroborada empiricamente. Se existe ajuste, ele não é significativo ou não alivia a restrição, pelo menos em um primeiro momento (Thirlwall, 2011). Assumindo então os termos de troca constantes, obtemos a regra simples de Thirlwall:

$$
\hat{y}=\frac{\Phi \hat{z}}{\pi}
$$

Dada a equação (8), o crescimento de longo prazo de uma economia é diretamente proporcional ao produto entre variação da renda externa e à razão entre as elasticidades renda-demanda das exportações e importações. O crescimento da renda interna é restringido pela elasticidade-renda das importações na medida em que há um limite de oferta de divisas com que essa economia pode contar para satisfazer suas necessidades de importar. Isso significa que, quanto maior a razão entre as elasticidades renda do comércio exterior de uma determinada economia, menor a restrição ao crescimento por parte do BP. Em economias primário-exportadoras, a tendência de crescimento é menor que a dos países industrializados na medida em que essa razão é menor para as primeiras² (Gouvêa; Lima, 2010). O gap de renda entre países desenvolvidos e subdesenvolvidos deriva dessa restrição.

A ideia de que, no longo prazo, o crescimento econômico é limitado pela emergência de desequilíbrios comerciais tem forte tradição na América Latina. Os trabalhos da Comissão Econômica para a América Latina e o Caribe (Cepal) e especialmente do economista argentino Raúl Prebisch

1 Na versão neoclássica, $\left(\widehat{P}_{d}-\widehat{P}_{f}\right)=\frac{\hat{y} \pi-\Phi \hat{z}}{(1+\varphi+\beta)}$, onde os preços se ajustam aos desequilíbrios no BP. Krugman (1989), assumindo paridade do poder de compra (PPP), desenvolve de forma independente um modelo em que $\frac{\hat{y}}{\hat{z}}=\frac{\Phi}{\pi}$, sendo que as elasticidades renda das exportações e importações se ajustariam às diferenças entre as taxas de crescimento.

2 Gouvêa e Lima (2010) estimaram a elasticidade renda da pauta exportadora brasileira por setores para o período 1962-2006, o que nos permite uma comparação bastante útil. Os coeficientes estimados para os produtos primários, intensivos em mão de obra/recursos naturais e de baixo conteúdo tecnológico foram de 1.14, 1.35 e 1.53, respectivamente. Já os setores de intensidade tecnológica média e alta apresentaram elasticidades de 2.16 e 2.96 . 
já apontavam o baixo dinamismo do setor exportador e a elevada propensão a importar como principal limitante do crescimento nas economias periféricas (Dávila-Fernández; Amado, 2015). Embora tenha desenvolvido seu modelo de forma independente, Thirlwall (1983) menciona as similaridades entre a sua formulação e a de Prebisch. No entanto, diferentemente da generalização de Thirwall, Prebisch argumentava que as restrições ao crescimento no BP eram impostas apenas para os países mais periféricos, e não aos do centro (Boianovsky; Solís, 2014).

A literatura de crescimento BOPC tem tomado dois caminhos distintos em termos dos exercícios de modelagem. Por um lado, autores como Araújo e Teixeira (2004) e Araújo e Lima (2007) têm feito importantes contribuições no sentido de incorporar o componente "mudança estrutural" a esse referencial teórico. Um segundo grupo de autores vem se esforçando para introduzir os demais elementos do Balanço de Pagamentos ao modelo original. Uma das hipóteses do artigo seminal de Thirlwall era que o equilíbrio na balança comercial equivale ao equilíbrio no Balanço de Pagamentos. Essa hipótese será relaxada permitindo aumento do grau de sofisticação do exercício e seu poder de representação do mundo real. Nossa contribuição se encaixa dentro das realizadas pelo segundo grupo.

O modelo teórico desenvolvido diferencia o investimento externo direto do investimento em carteira na determinação da taxa de crescimento compatível com a restrição externa, de modo que parte do fluxo de capitais decorre do diferencial da taxa de juros doméstica, internacional e do risco país. O interesse em separar o IED do IC é particularmente maior no estudo da dinâmica de economias em desenvolvimento, como a brasileira.

O exercício proposto será dividido em dois momentos. Inicialmente, o investimento em carteira será tomado como função do diferencial da taxa de juros doméstica, internacional e do risco país. Quando a incerteza (não probabilística) aumenta no sistema financeiro internacional, quer em relação à economia mundial, quer em relação à determinada economia, mesmo um diferencial de taxas de juros positivo pode não ser suficiente para elevar o influxo líquido de capitais no BP dessa economia. A explicação está no aumento do risco país. Essa assertiva é confirmada pela ocorrência de crises cambiais que não puderam ser contidas com a elevação do citado diferencial de juros, tais como a crise do México (1994), da Ásia (1997), da Rússia (1998) ou do Brasil (1999). Com base nessa formulação, faremos a derivação completa da taxa de crescimento que equilibra o Balanço de Pagamentos. 
Em um segundo momento, tomamos o risco país como igual a zero e constante de modo que o IC dependa apenas do diferencial de juros. Esse formato será bastante útil por permitir melhor visualização da dinâmica entre o crescimento de longo prazo e o comportamento da taxa de juros doméstica. Todavia, deixamos claro desde já que a atração do investimento em carteira também é afetada pelo humor e pelas mudanças de expectativas no sistema financeiro internacional, o que explica a razão para a ocorrência de crises cambiais, mesmo no contexto de aumento do diferencial de juros interno e externo.

\subsection{Incorporando fluxo de capitais}

Embora bastante prática, a expressão (8) não leva em consideração um componente importante do BP, especialmente para países em desenvolvimento como o Brasil: as contas capital e financeira. Com o objetivo de tornar o modelo mais fidedigno, Thirlwall e Hussain (1982) 3 incorporam a CCF à equação (3), de modo que ela assume a seguinte forma:

$$
P_{d} X+P_{d} C=P_{f} M
$$

em que $C$ corresponde ao valor do fluxo de capitais, sendo $C>0$, se houver entrada de capital, e $C<0$, caso haja uma saída de capital. Logaritmando e derivando no tempo:

$$
\theta_{1}\left(\widehat{p_{d}}+\hat{x}\right)+\theta_{2}\left(\widehat{p_{d}}+\hat{c}\right)=\widehat{P_{f}}+\hat{m}
$$

onde $\theta_{1}=\frac{P_{d} X}{P_{f} M}$ e $\theta_{2}=\frac{P_{d} C}{P_{f} M}$ representam a fração das exportações e do fluxo de capitais como proporção das importações e $\theta_{1}+\theta_{2}=1$. Note que $\theta_{1}$ e $\theta_{2}$ não são coeficientes fixos que variam de acordo com as mudanças na proporção das exportações e do fluxo de capitais cobertos pelas importações. Todavia, para efeito de simplificação, eles serão tomados como constantes a menos que seja indicado o contrário. Substituindo (4) e (5) em (10) e isolando $\hat{y}$ :

3 Nesta representação consideramos o fluxo de capitais igual a $P_{d} C$ embora em sua versão original C não estivesse ponderado pelo nível de preços. Esta alteração não modifica os resultados originais e nos permite uma melhor visualização da expressão final. 


$$
\hat{y}=\frac{\left(1+\theta_{1} \varphi+\beta\right)\left(\widehat{p_{d}}-\widehat{p_{f}}\right)+\theta_{1} \Phi \hat{z}+\theta_{2} \hat{c}}{\pi}
$$

Assumindo os termos de troca constantes obtemos:

$$
\hat{y}=\frac{\theta_{1} \Phi \hat{z}+\theta_{2} \hat{c}}{\pi}
$$

Esta nova expressão nos permite observar o impacto do fluxo de capitais sobre a restrição no Balanço de Pagamentos. $O$ investimento externo, seja ele direto, seja ele indireto, alivia essa restrição e permite maior taxa de crescimento. Entretanto, nessas condições, uma economia poderia se financiar ilimitadamente com poupança externa, o que não ocorre na prática. Buscando superar esse problema, Moreno-Brid (1999) propõe que a razão entre o fluxo de capitais $\mathrm{C}$ e o produto doméstico $\mathrm{Y}$ seja constante. Desse modo:

$$
\frac{P_{d} C}{P_{d} Y}=k
$$

onde k é uma constante e representa o limite máximo que essa economia consegue se endividar como proporção do PIB. A equação (13) é equivalente a:

$$
\widehat{p_{d}}+\hat{c}=\widehat{p_{d}}+\hat{y}
$$

Moreno-Brid (2003) adiciona ainda o pagamento de juros decorrentes da entrada de capitais. Assim:

$$
L=P_{d} R_{d}^{\omega}
$$

sendo que L corresponde ao pagamento de juros, $R_{d}$ representa a taxa de juros, $\omega$ é a elasticidade de $L$ em relação a $R_{d}$, e o nível de preços é dado por $P_{d}$. Logaritmando e derivando em relação ao tempo, temos:

$$
\hat{l}=\widehat{p_{d}}+\omega \widehat{r_{d}}
$$


Incorporando essas modificações, o modelo passa a possuir os principais elementos que compõem efetivamente o BP. O equilíbrio no Balanço de Pagamentos pode ser escrito então como:

$$
P_{d} X+P_{d} C-L=P_{f} M
$$

Empregando o mesmo ferramental utilizado até aqui, podemos reescrever essa nova expressão em termos das taxas de variação como:

$$
\theta_{1}\left(\widehat{p_{d}}+\hat{x}\right)+\theta_{2}\left(\widehat{p_{d}}+\hat{c}\right)-\theta_{3} \hat{l}=\widehat{p_{f}}+\hat{m}
$$

em que $\theta_{1}=\frac{P_{d} X}{P_{f} M}, \theta_{2}=\frac{P_{d} C}{P_{f} M}$ e $\theta_{3}=\frac{L}{P_{f} M}$ representam a fração das exportações, do fluxo de capitais e do pagamento de juros como proporção das importações, respectivamente, e $\theta_{1}+\theta_{2}-\theta_{3}=1$. Solucionando o sistema formado pelas equações (4), (5), (14), (16) e (18):

$$
\hat{y}=\frac{\left(1+\theta_{1} \varphi+\beta\right)\left(\widehat{p_{d}}-\widehat{p_{f}}\right)+\theta_{1} \Phi \hat{z}-\theta_{3} \omega \widehat{r_{d}}}{\pi-\theta_{2}}
$$

Mantendo os termos de troca constantes, obtemos a seguinte expressão para a taxa de crescimento em equilíbrio:

$$
\hat{y}=\frac{\theta_{1} \Phi \hat{z}-\theta_{3} \omega \hat{r_{d}}}{\pi-\theta_{2}}
$$

Da mesma forma que na Lei deThirlwall original, a taxa de crescimento do produto é diretamente proporcional ao crescimento da renda externa. Por outro lado, como era de se esperar, o pagamento de juros opera no sentido de restringir o crescimento.

Em steady-state a taxa de juros não pode crescer ou decrescer indefinidamente, i.e. $\widehat{r_{d}}=0$. Considerando ainda que $\theta_{1}=1-\theta_{2}+\theta_{3}$, a expressão acima pode ser reescrita como:

$$
\hat{y}=\frac{\left(1-\theta_{2}+\theta_{3}\right) \Phi \hat{z}}{\pi-\theta_{2}}
$$


$O$ leitor pode facilmente constatar que $\frac{\partial \hat{y}}{\partial \theta_{2}}<0$. Isso ocorre porque na realidade uma maior entrada de poupança externa reduz $\theta_{1}$. Daí que a simples absorção de poupança externa sem mudança estrutural reduz a taxa de crescimento da economia de longo prazo.

Sabendo da importância que a conta de "Transferências" tem no fechamento do BP em economias subdesenvolvidas no Caribe e no continente africano, Alleyne e Francis (2008) propõem que a equação (17) incorpore esse componente. Desse modo, seja:

$$
T=P_{d}\left(\frac{Z}{Y}\right)^{\gamma}
$$

em que $T$ corresponde às transferências unilaterais e é modelado em função da renda externa $Z$, da renda interna $Y$ e da elasticidade das transferências $\gamma$ em relação a $Z$ e $Y$, sendo $\gamma>0$. Dado um aumento da renda do resto do mundo, é de se esperar um aumento das transferências. Analogamente, um aumento da renda doméstica reduz o volume transferido. Logaritmando e derivando em relação ao tempo:

$$
\hat{t}=\widehat{p_{d}}+\gamma(\hat{z}-\hat{y})
$$

A nova expressão de equilíbrio no Balanço de Pagamentos pode ser escrita como:

$$
P_{d} X+P_{d} C-L+T=P_{f} M
$$

Reescrevendo a equação acima em função das taxas de variação, obtemos:

$$
\theta_{1}\left(\widehat{p_{d}}+\hat{x}\right)+\theta_{2}\left(\widehat{p_{d}}+\hat{c}\right)-\theta_{3} \hat{l}+\theta_{4} \hat{t}=\widehat{p_{f}}+\hat{m}
$$

com $\theta_{1}, \theta_{2}$ e $\theta_{3}$ representando a fração das exportações, do fluxo de capitais e do pagamento de juros como proporção das importações, $\theta_{4}=\frac{T}{P_{f} M}$ sendo a proporção das transferências em relação às importações e $\theta_{1}+\theta_{2}-\theta_{3}+\theta_{4}=1$. Resolvendo o sistema formado pelas equações (4), (5), (14), (16), (22) e (24): 


$$
\hat{y}=\frac{\left(1+\theta_{1} \varphi+\beta\right)\left(\widehat{p_{d}}-\widehat{p_{f}}\right)+\left(\theta_{1} \Phi+\gamma \theta_{4}\right) \hat{z}-\theta_{3} \omega \widehat{r_{d}}}{\pi-\theta_{2}+\theta_{4} \gamma}
$$

Fazendo os termos de troca constantes, obtemos a seguinte expressão para a taxa de crescimento em equilíbrio:

$$
\hat{y}=\frac{\left(\theta_{1} \Phi+\gamma \theta_{4}\right) \hat{z}-\theta_{3} \omega \hat{r_{d}}}{\pi-\theta_{2}+\theta_{4} \gamma}
$$

Em termos de resultados, Alleyne e Francis (2008) mantêm essencialmente todos os anteriores. Sua contribuição, no entanto, permite obter uma expressão que contém todos os elementos do BP, sendo mais fidedigna a ele do ponto de vista teórico. $O$ efeito de um aumento das transferências sobre o crescimento pode ser positivo ou negativo a depender da combinação de parâmetros da economia.

Os modelos de crescimento balance-of-payments-constrained têm sido corroborados empiricamente em uma série de estudos tanto em países desenvolvidos quanto em desenvolvimento. Thilrwall (1979), no artigo em que desenvolveu a lei que leva seu nome, testou sua formulação para o período 1953-1976 em uma amostra de 18 países. O autor encontrou estimativas para a taxa de crescimento compatíveis com a taxa de crescimento efetiva.

Thirlwall e Hussain (1982), sabendo da importância que fluxos financeiros têm em economias em desenvolvimento, estenderam o exercício original incorporando os fluxos financeiros para 15 países, entre 1951-1969. A conclusão de sua análise indica que o movimento de capitais foi significativo na determinação da taxa de crescimento compatível com o equilíbrio no BP. Ademais, encontra-se que mudanças nos preços relativos foram estatisticamente significativas para alguns países da amostra.

Alonso e Garcimartín (1999), utilizando data para um grupo de 10 países-membros da OCDE, entre 1965-1994, mostram que a Lei de Thirlwall se sustenta empiricamente e que os preços relativos não têm papel significativo em termos de crescimento econômico. Os resultados encontrados corroboram a posição keynesiana, não havendo evidência significativa de ajuste nos preços.

Romero, Silveira e Jayme Jr. (2011) encontram que o modelo de Thirlwall simples e em sua versão multissetorial explicam com sucesso o crescimento econômico brasileiro nos últimos cinquenta anos. Os autores 
empregam três métodos distintos de estimação para os anos 1962 a 2007. Gouvea e Lima (2010) testam a Lei de Thirlwall multissetorial para uma amostra de oito países - quatro asiáticos e quatro latino-americanos - entre 1962 e 2006, encontrando que não se pode rejeitar a hipótese de que o crescimento desses países é restringido pelo Balanço de Pagamentos.

Utilizando amostras maiores, Cimoli, Porcile e Rovira (2010) realizam um exercício econométrico verificando a aplicabilidade da Lei de Thirlwall para 29 países - oito latino-americanos, 15 membros da OCDE e seis asiáticos. O período analisado compreende os anos de 1961 a 2004. Ademais do suporte encontrado a favor da formulação de Thirlwall, mostra-se que a evolução das elasticidades das importações e exportações no tempo entre os países latino-americanos e asiáticos é capaz de explicar a trajetória divergente de suas rendas per capita.

Bagnai (2010) além de uma extensa revisão da literatura empírica sobre a lei de Thirlwall testa validade da teoria para uma amostra de 22 países membros da OCDE entre 1960 e 2006. O autor utiliza técnicas de cointegração que permitem a presença de quebras estruturais endógenas nos parâmetros de longo prazo. O trabalho não apenas confirma a lei de Thirlwall como mostra que ao permitir quebras estruturais o modelo aumenta o grau de aderência das estimativas aos valores reais.

Por fim, em um esforço bastante ambicioso, Gouvea e Lima (2013) testam a versãomultissetorial da Lei de Thirlwall para uma amostra de 90 economias entre 1965 e 1999. O poder preditivo do modelo é testado comparando a taxa de crescimento prevista com a taxa de crescimento efetiva. Apesar de, como esperado, o crescimento de todos os países não é restrito pelo Balanço de Pagamentos, a "curva de $45^{\circ}$ " não pode ser rejeitada a um nível de significância de 5\%, o que dá suporte à lei.

\section{Separando o IED do investimento em carteira}

As contribuições revisitadas de Moreno-Brid $(1999,2003)$ e Alleyne e Francis (2008) permitem derivar a taxa de crescimento compatível com a restrição externa com base em uma abordagem mais sofisticada e com maior aderência ao mundo real. Todavia, elas não são capazes de captar o efeito do diferencial das taxas de juros doméstica e externa sobre o fluxo de capitais e consequentemente sobre a taxa de crescimento que equilibra o BP. 
O interesse em separar o IED do IC é particularmente maior no estudo da dinâmica de economias em desenvolvimento.

O Gráfico 1 nos permite observar o comportamento da taxa básica de juros da economia brasileira na última década. Depois de manter uma trajetória ascendente nos dois últimos anos do governo FHC e no primeiro ano do governo Lula, a taxa SELIC tem apresentado tendência de queda tanto em termos nominais quanto em reais. Entretanto, em quase todo o período analisado, ela se mantém muito acima dos $5 \%$, já descontada a inflação esperada. Para efeito de comparação, nos países desenvolvidos a taxa média real está entre 1\% e 3\%, ao passo que, nos países em desenvolvimento, ela oscila entre 3\% e 5\% (Bresser-Pereira; Nakano, 2002).

\section{Gráfico 1 Taxa SELIC anualizada, 2000-2013 (em porcentagens)}

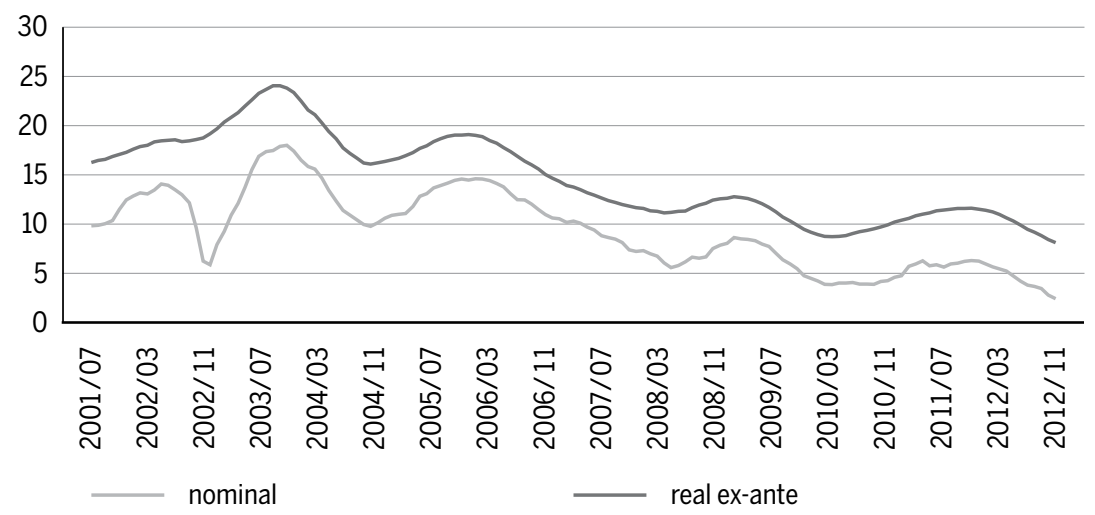

Fonte: Ipea data; elaborado pelo autor.

Procurando melhor entender o efeito da taxa de juros sobre o crescimento de longo prazo, reformulamos a equação que representa o fluxo de capitais (C), desagregando-a em duas novas:

$$
\begin{aligned}
& I E D=P_{d} Y^{\tau} \\
& I C=P_{d}\left(R_{d}-R_{f}-R_{p}\right)^{\alpha}
\end{aligned}
$$

em que $R_{d}$ corresponde à taxa de juros interna; $R_{f}$, à taxa de juros externa; e $R_{p}$, ao risco país. Por sua vez, $\alpha>0$ corresponde à elasticidade de $I C$ em 
relação à diferença entre as taxas de juros doméstica/internacional/risco, e $\tau>0$ equivale à elasticidade de $I E D$ em relação à renda.

Dentro da conta "Capital e Financeira", o componente de investimento externo direto (IED) responde a variações na renda interna. É natural pensar que um aumento da renda doméstica atrai investimentos produtivos de médio e longo prazos. Se pensarmos em termos de investimento em carteira (IC), a taxa de juros passa a ser uma variável importante para captação de poupança externa. Efetivamente, ela foi usada explicitamente com esse objetivo durante a estabilização dos anos 1990 (Bresser-Pereira; Nakano, 2002; Hermann, 2010).

A atração de capitais externos é proporcionada pelo diferencial de taxas de juros interna e externa menos o risco país. O risco país não pode ser considerado como um parâmetro, mas, sim, como uma variável. Quando a incerteza (não probabilística) aumenta no sistema financeiro internacional, mesmo um diferencial de taxas de juros interna e externa positivo pode não ser suficiente para elevar o influxo líquido de capitais no BP dessa economia. A explicação está no aumento do risco país. Essa assertiva é confirmada pela ocorrência de crises cambiais que não puderam ser contidas com a elevação do citado diferencial de juros, tais como a crise do México (1994), da Ásia (1997), da Rússia (1998) ou do Brasil (1999).

Logaritmando e derivando em função do tempo, as equações (27) e (28) assumem o seguinte formato:

$$
\begin{aligned}
& \widehat{i e d}=\widehat{p_{d}}+\tau \hat{y} \\
& \hat{i c}=\widehat{p_{d}}+\alpha\left(\rho_{1} \widehat{r_{d}}-\rho_{2} \widehat{r_{f}}-\rho_{3} \widehat{r_{p}}\right)
\end{aligned}
$$

com $\rho_{1}=\frac{R_{d}}{R_{d}-R_{f}-R_{p}}, \rho_{2}=\frac{R_{f}}{R_{d}-R_{f}-R_{p}} \quad$ e $\rho_{3}=\frac{R_{p}}{R_{d}-R_{f}-R_{p}}$ correspondendo à proporção dos juros internos, externos e do risco país em relação à diferença entre eles. Nessa representação, assumimos por hipótese que $R_{d}>R_{f}+R_{p}$. Perceba que, para um $R_{f}+R_{p}$ fixo, $\lim _{R_{d} \rightarrow \infty} \rho_{1}=1$ e $\frac{\delta \rho_{1}}{\delta R_{d}}<0$ (ver Apêndice 1). Isso implica que o efeito de um aumento da taxa de juros doméstica sobre o fluxo de capitais de curto prazo é decrescente no tempo. Como a conta "Transferências" corresponde a valores desprezíveis em termos do BP brasileiro, reescrevemos o equilíbrio no Balanço de Pagamentos como: 


$$
P_{d} X+I E D+I C-L=P_{f} M
$$

Em termos de taxas de variação, a equação acima equivale a:

$$
\theta_{1}\left(\widehat{p_{d}}+\hat{x}\right)+\theta_{2} \widehat{i e d}+\theta_{3} \hat{i c}-\theta_{4} \hat{l}=\widehat{p_{f}}+\hat{m}
$$

em que $\theta_{1}=\frac{P_{d} X}{P_{f} M}, \theta_{2}=\frac{I E D}{P_{f} M}, \theta_{3}=\frac{I C}{P_{f} M}{ }^{\mathrm{e}} \theta_{4}=\frac{L}{P_{f} M}$ representam a fração das exportações, do IED, do IC e do pagamento de juros como proporção das importações, respectivamente. Finalmente $\theta_{1}+\theta_{2}+\theta_{3}-\theta_{4}=1$. Substituindo as equações (4), (5), (16), (29) e (30) em (32) e resolvendo para $\hat{y}$ :

$$
\hat{y}=\frac{\left(1+\theta_{1} \varphi+\beta\right)\left(\widehat{p_{d}}-\widehat{p_{f}}\right)+\theta_{1} \Phi \hat{z}+\left(\theta_{3} \alpha \rho_{1}-\theta_{4} \omega\right) \widehat{r_{d}}-\theta_{3} \alpha\left(\rho_{2} \hat{r_{f}}+\rho_{3} \widehat{r_{p}}\right)}{\pi-\theta_{2} \tau}
$$

Assumindo termos de troca constantes, temos nossa versão final da Lei de Thirlwall com fluxo de capitais:

$$
\hat{y}=\frac{\theta_{1} \Phi \hat{z}+\left(\theta_{3} \alpha \rho_{1}-\theta_{4} \omega\right) \hat{r_{d}}-\theta_{3} \alpha\left(\rho_{2} \hat{r_{f}}+\rho_{3} \hat{r_{p}}\right)}{\pi-\theta_{2} \tau}
$$

A relação entre as taxas de crescimento interna e externa é mantida, da mesma forma que sua relação com as elasticidades renda-demanda das exportações e importações. De igual modo, é possível observar que, para um aumento na taxa de variação da taxa de juros externa ou do risco país, há um efeito negativo sobre a taxa de crescimento ${ }^{4}$. Doravante, merece atenção o efeito da taxa de juros interna sobre a restrição ceterisparibus:

$$
\frac{\delta \hat{y}}{\delta \hat{r}_{d}}=\frac{\theta_{3} \alpha \rho_{1}-\theta_{4} \omega}{\pi-\theta_{2} \tau}
$$

Seguindo Moreno-Brid (2003) e Alleyne e Francis (2008), seja o denominador da equação positivo, $\pi-\theta_{2} \tau>0$. Se $\theta_{3} \alpha \rho_{1}>\theta_{4} \omega$, um aumento de $\hat{r_{d}}$ alivia a restrição sobre o Balanço de Pagamentos, aumentando as-

4 Notemos entretanto que toda a análise subsequente ocorre fora do steady-state, quando $\hat{r_{d}}, \hat{r_{f}}$ e $\hat{r}_{p}$ são diferentes de zero. 
sim a taxa de crescimento potencial de longo prazo. Por outro lado, se $\theta_{3} \alpha \rho_{1}<\theta_{4} \omega$, o aumento de $\widehat{r_{d}}$ reduzirá a taxa de crescimento compatível com o equilíbrio no Balanço de Pagamentos. Para $\theta_{3} \alpha \rho_{1}=\theta_{4} \omega$, o efeito sobre $\hat{y}$ é nulo. Notemos, no entanto, que a relação entre esses dois componentes não é constante, e sua dinâmica é determinada por $\rho_{1}$. Como para um $R_{f}$ e $R_{p}$ fixos, $\lim _{R_{d} \rightarrow \infty} \rho_{1}=1$ e $\frac{\delta \rho_{1}}{\delta R_{d}}<0$, o efeito de um aumento da taxa de juros doméstica sobre o fluxo de capitais de curto prazo é decrescente no tempo.

Façamos apenas, a título de simplificação, $R_{p}$ constante e igual a zero de modo que o investimento em carteira seja função apenas do diferencial da taxa de juros. $O$ Gráfico 2 permite observarmos a dinâmica entre $\rho_{1}$ e $R_{d}$ :

\section{Gráfico 2 Dinâmica entre $\rho_{1}$ e $R_{d}$}

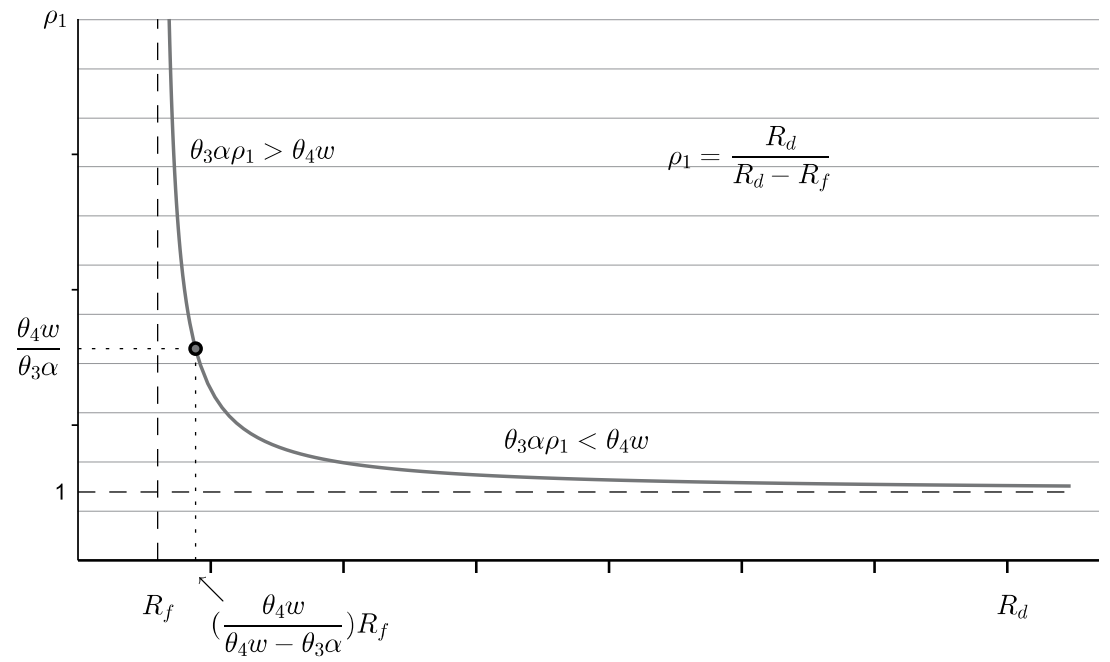

Fonte: Elaborado pelo autor.

Isolando $\rho_{1}$ em $\theta_{3} \alpha \rho_{1}>\theta_{4} \omega$, obtemos:

$$
\rho_{1}=\frac{\theta_{4} \omega}{\theta_{3} \alpha}
$$

Substituindo $\rho_{1}=\frac{R_{d}}{R_{d}-R_{f}}$ na expressão (36), encontramos a relação entre as taxas de juros doméstica e externa que não altera a restrição do BP: 


$$
R_{d}=\left(\frac{\theta_{4} \omega}{\theta_{4} \omega-\theta_{3} \alpha}\right) R_{f}
$$

Como tanto $R_{d}$ quanto $R_{f}$ são valores positivos, devemos impor uma restrição de equilíbrio de modo que $\theta_{4} \omega-\theta_{3} \alpha>0$. Isso significa que a elasticidade da remessa de lucros ao exterior em relação à taxa de juros interna, $\omega$, ponderada pelo peso da conta $\mathrm{SR}, \theta_{4}$, deve ser necessariamente superior à elasticidade do investimento em carteira em relação ao diferencial de juros, $\alpha$, ponderado pelo peso do IC.

Como podemos observar no Gráfico 2, para um $R_{d} \geq\left(\frac{\theta_{4} \omega}{\theta_{4} \omega-\theta_{3} \alpha}\right) R_{f}{ }^{\prime}$ aumentos da taxa de juros interna aumentam a restrição ao crescimento no Balanço de Pagamentos. Isso significa que um aumento da taxa de juros doméstica reduz o crescimento potencial da economia enquanto uma redução de juros é favorável ao crescimento. Por outro lado, se $R_{f}<R_{d}<\left(\frac{\theta_{4} \omega}{\theta_{4} \omega-\theta_{3} \alpha}\right) R_{f}$ um aumento dos juros alivia a restrição sobre o $\mathrm{BP}$ ao passo que sua redução tem o efeito oposto.

Há uma tensão intrínseca entre a taxa de juros que capta a poupança externa e a que determina o pagamento de "Serviços e Rendas". Descrevendo o mesmo fenômeno agora em termos de $\rho_{1}$, quando $\rho_{1}>\frac{\theta_{4} h}{\theta_{3} \alpha}$, a poupança externa "extra" captada pelo aumento dos juros é maior que o valor a ser pago em termos de serviço da dívida. Daí o efeito positivo de um aumento dos juros sobre a taxa de crescimento compatível com o BP. Quando $\rho_{1}<\frac{\theta_{4} h}{\theta_{3} \alpha}$, temos o efeito contrário.

Façamos agora um exercício de estática comparativa para estudar o efeito de variações na taxa de juros internacional ou do risco país sobre a restrição externa. Temos então $\frac{\delta \hat{y}}{\delta \widehat{r_{f}}}=\frac{-\theta_{3} \alpha \rho_{2}}{\pi-\theta_{2} \tau}$ e $\frac{\delta \hat{y}}{\delta \hat{r}_{p}}=\frac{-\theta_{3} \alpha \rho_{3}}{\pi-\theta_{2} \tau}$. Em ambos os casos, o sinal da derivada parcial é negativo. Na fase ascendente do ciclo de liquidez internacional, a combinação de uma redução dos juros internacionais e do risco país tem impacto positivo sobre a taxa de crescimento de equilíbrio do BP. Por outro lado, na fase descendente, o efeito contrário prevalece pela combinação de uma elevação dos juros e do risco país. 
Embora não seja responsabilidade direta da autoridade monetária, a modificação da taxa básica de juros pode repercutir positiva ou negativamente sobre o crescimento de longo prazo. Cremos ser de vital importância que os policymakers deem maior atenção aos efeitos da política monetária e financeira sobre a atividade econômica não apenas de curto, mas de longo prazo.

\section{Considerações finais}

Este trabalho revisou o modelo básico de crescimento com restrição no Balanço de Pagamentos e suas principais modificações, procurando esclarecer alguns aspectos dos modelos de crescimento com restrição nesse balanço. Formalizamos um modelo teórico em que o investimento externo direto é diferenciado do investimento em carteira na determinação da taxa de crescimento que equilibra o BP. Desse modo, parte da atração de capitais externos decorre do diferencial de taxas de juros doméstica e externa.

Pensando na importância que o diferencial de juros tem para economias em desenvolvimento como a brasileira, introduzimos uma modificação no modelo de Moreno-Brid (2003) que nos permitisse melhor visualizar seus efeitos sobre o Balanço de Pagamentos. A expressão final encontrada foi:

$$
\hat{y}=\frac{\theta_{1} \Phi \hat{z}+\left(\theta_{3} \alpha \rho_{1}-\theta_{4} \omega\right) \hat{r_{d}}-\theta_{3} \alpha\left(\rho_{2} \hat{r_{f}}+\rho_{3} \hat{r_{p}}\right)}{\pi-\theta_{2} \tau}
$$

A relação entre as taxas de crescimento interna e externa, em comparação com a dos modelos originais, foi mantida, da mesma forma que sua relação com as elasticidades renda-demanda das exportações e importações. Todavia, no tocante à variação na taxa de juros interna, o efeito é ambíguo. Caso a elasticidade do investimento em carteira em relação ao diferencial de juros e o risco país, ponderado pelo peso do IC no BP, for maior que a elasticidade da remessa de lucros ao exterior ponderado pelo seu respectivo peso no BP, então um aumento da taxa de juros doméstica contribui positivamente para o crescimento de longo prazo. Caso contrário, o efeito sobre $\hat{y}$ será negativo.

Realizando as devidas manipulações algébricas, encontramos a relação entre as taxas de juros doméstica e externa que não altera a restrição do BP: 


$$
R_{d}=\left(\frac{\theta_{4} \omega}{\theta_{4} \omega-\theta_{3} \alpha}\right) R_{f}
$$

Enquanto $R_{d} \geq\left(\frac{\theta_{4} \omega}{\theta_{4} \omega-\theta_{3} \alpha}\right) R_{f}$, aumentos da taxa de juros interna aumentam a restrição ao crescimento no Balanço de Pagamentos. Isso significa que, mantendo-se a desigualdade acima, um aumento dos juros internos compromete a taxa de crescimento compatível com o equilíbrio na BP. Por outro lado, se $R_{f}<R_{d}<\left(\frac{\theta_{4} \omega}{\theta_{4} \omega-\theta_{3} \alpha}\right) R_{f}$, um aumento dos juros alivia a restrição sobre o BP. Isso ocorre porque há uma tensão intrínseca entre a taxa de juros que capta o investimento em carteira e a que determina o pagamento de "Serviços e Rendas".

Dito isso, concluímos que, dado o efeito dúbio que variações da taxa de juros têm sobre o crescimento compatível com equilíbrio no BP, os policymakers devem dar maior atenção aos efeitos das políticas monetária e financeira sobre a atividade econômica não apenas de curto, mas de longo prazo. $O$ modelo desenvolvido permite ter uma visão mais abrangente e com maior poder de representação do mundo real, incorporando de forma mais adequada os elementos do BP ao referencial teórico.

\section{Referências}

ALLEYNE, D.; FRANCIS, A. A. Balance of payments-constrained growth in developing countries: A theoretical perspective. Metroeconomica, vol.59, n. 2, p. 189-202, 2008.

ALONSO, J. A.; GARCIMARTÍN, C. A new approach to balance-of-payments constraint: Some empirical evidence. Journal of Post Keynesian Economics, vol. 21, n. 2, p. 259-281, 1999.

ARAÚJO, R. A.; LIMA, G. T.A structural economic dynamics approach to balance-of-payments-constrained growth. Cambridge Journal of Economics, v. 31, p. 755-774, 2007.

ARAÚJO, R. A.; TEIXEIRA, J. A Pasinettian approach to international economic relations: The pure labour case. Review of Political Economy, vol. 16, n. 1, p. 117-129, 2004.

BAGNAI, A. Structural breaks, cointegration, and the empirics of Thirlwall's Law. Applied Economics vol. 42, p. 1315-1329, 2010.

BRESSER-PEREIRA, L. C.; NAKANO, Y. Uma estratégia de desenvolvimento com estabilidade. Revista de Economia Política, vol. 22, n. 3, 2002.

BOIANOVSKY, M.; SOLÍS, R. The origins and development of the Latin American structuralist approach to the Balance of Payments, 1944-1964. Review of Political Economy, v.26, n.1, p.23-59, 2014. 
CIMOLI, M.; PORCILE, G.; ROVIRA, S. Structural change and the BOP-constraint: Why did Latin America fail to converge? Cambridge Journal of Economics, vol. 34, p. 389-411, 2010.

DÁVILA-FERNÁNDEZ, M. J.; AMADO, A. Entre a lei de Thirlwall e a hipótese Prebisch-Singer: uma avaliação da dinâmica dos termos de troca em um modelo de crescimento com restrição no Balanço de Pagamentos. Economia e Sociedade, vol. 24, n.1, p. 87-119, 2015.

GOUVEA, R. R.; LIMA, G. Structural change, balance-of-payments constraint, and economic growth: Evidence from the multisectoral Thirlwall's Law. Journal of Post Keynesian Economics, vol. 33, n. 1, p. 169-204, 2010.

GOUVEA, R. R.; LIMA, G. Balance of payments constrained growth in a multisectoral framework: A panel data investigation. Journal of Economic Studies, vol. 40, n. 2, p. 240-254, 2013.

HERMANN, J. Liberalização e desenvolvimento financeiro: Lições da experiência brasileira no período 1990-2006. Economia e Sociedade, v. 19, p. 257-290, 2010.

KRUGMAN, P. Differences in income elasticities and trends in real exchange rates. European Economic Review, vol. 33, p. 1031-1054, 1989.

MORENO-BRID, J. C. On capital flows and the balance-of-payments-constrained growth model. Journal of Post Keynesian Economics, vol. 21, n. 2, p. 283-297, 1999.

MORENO-BRID, J. C. Capital flows, interest payments and the balance-of-payments constrained growth model: A theoretical and empirical analysis. Metroeconomica, vol. 54, n. 2, p. 356-365, 2003.

ROMERO, J. P.; SILVEIRA, F.; JAYME JR, F. G. Brasil: Cambio estructural y crecimiento con restricción de balanza de pagos. Revista Cepal, n. 105, 2011.

SETTERFIELD, M. The remarkable durability of Thirlwall's Law. PSL Quarterly Review, v. 64, n. 2, p. 393-427, 2011a.

SETTERFIELD, M. Post-Keynesian macrodynamics and path-dependent growth. European Journal of Economics and Economic Policies: Intervention, vol. 8, n. 2, p. 299-316, 2011 b.

THIRLWALL, A. P. The balance of payments constraint as an explanation of international growth rate differences. BNL Quarterly Review, vol. 32, p. 45-53, 1979.

THIRLWALL, A. P. Foreign trade elasticities in centre-periphery models of growth and development. BNL Quarterly Review, v. 36, p. 249-61, 1983.

THIRLWALL, A. P. Balance of payments constrained growth models: History and overview. PSL Quarterly Review, vol. 64, n. 259, p. 307-351, 2011.

THIRLWALL, A. P.; HUSSAIN, M. N. The balance of payments constraint, capital flows and growth rate differences between developing countries. Oxford EconomicPapers, New Series, vol. 34, n 3, p. 498-510, 1982.

\section{Sobre o autor}

MarwilJ.Dávila-Fernández-marwil_davila@hotmail.com

$\mathrm{PhD}$ Candidate, Dipartimento di Economia Politica e Statistica,Università degli Studi di Siena, Italie.

\section{Sobre o artigo}

Recebido em $1^{\circ}$ de maio de 2013. Aprovado em 18 de fevereiro de 2015. 


\section{APÊNDICE}

Seja $\rho_{1}=\frac{R_{d}}{R_{d}-R_{f}-R_{p}}$, em que $R_{d}$ corresponde à taxa de juros interna, $R_{f}$ equivale à taxa de juros externa, e $R_{p}$, ao risco país:

$$
\lim _{R_{d} \rightarrow \infty} \rho_{1}=\lim _{R_{d} \rightarrow \infty} \frac{R_{d}}{R_{d}-R_{f}-R_{p}}=\lim _{R_{d} \rightarrow \infty} \frac{1}{\left(1-\frac{R_{f}+R_{p}}{R_{d}}\right)}=1
$$

Derivando ainda $\rho_{1}$ em relação à taxa de juros doméstica:

$$
\frac{\delta \rho_{1}}{\delta R_{d}}=\frac{\left(R_{d}-R_{f}-R_{p}\right)-R_{d}}{\left(R_{d}-R_{f}-R_{p}\right)^{2}}=\frac{-\left(R_{f}+R_{p}\right)}{\left(R_{d}-R_{f}\right)^{2}}<0
$$

